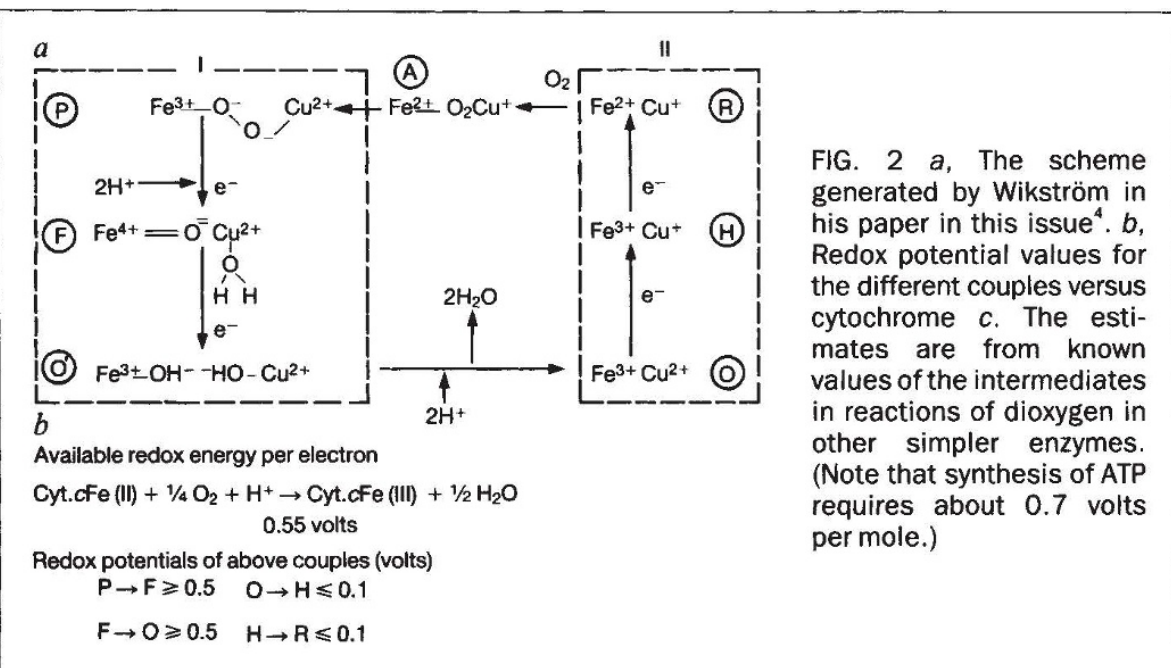

directly with dioxygen and which must diffuse through the protein matrix to the reaction centre in the membrane, additional (one $\mathrm{H}^{+}$per electron) protons are in fact energized by the reactions of molecular dioxygen in the membrane, and that these protons are pumped (in proton channels) across the membrane by gated energized diffusion ${ }^{3}$. The nature of the gating is as yet unknown. In an effort to elucidate the reaction path, Wikström now considers more precisely the coupling steps between the reduction of dioxygen by electrons and this proton pumping ${ }^{4}$. $\mathrm{He}$ concludes that proton pumping, which, note again, is separate from the protonation steps of dioxygen itself, occurs in association with the reaction steps involving only the first and second electrons of the four required to convert $\mathrm{O}_{2}$ to $2 \mathrm{H}_{2} \mathrm{O}$. I shall now consider some consequential mechanistic features.

In a protein such as cytochrome oxidase, consisting in all probability of membrane helices 5.6 , cooperativity should occur via conformational changes on $\mathrm{O}_{2}$ uptake or electron transfer as in haemoglobin and haemocyanin. In fact the $a_{3} \cdot \mathrm{Cu}_{\mathrm{B}}$ centre of the cytochrome oxidase can be thought of as half haemoglobin and half haemocyanin (Fig. 1). Now the reactions of these proteins and of oxidases such as cytochrome $P_{450}$ and even of cytochrome $c$ with ligands are known to occur from one side of the proteins only via groove-opening reactions. It is hardly surprising, then, that the reactions of cytochrome oxidase, $\mathrm{O}_{2}+4 e+4 \mathrm{H}^{+} \rightarrow 2 \mathrm{H}_{2} \mathrm{O}$, occur through the oxidase from only one side of the membrane in which the protein is lodged. The apparent surprise is that the additional proton pumping in a different, helical channel is driven by the first two steps of reduction only.

Let us consider the steps. Each of the steps $\mathrm{R} \rightarrow \mathrm{P}, \mathrm{P} \rightarrow \mathrm{F}, \mathrm{F} \rightarrow \mathrm{O}, \mathrm{O} \rightarrow \mathrm{H}$ and $\mathrm{H} \rightarrow \mathrm{R}$ are expected to be linked to conformational changes (Fig. 2 and see Fig. 1). The step $\mathbf{R} \rightarrow \mathbf{P}$ is not reversible. Whatever the series of conformational switches, the later steps return the protein to its initial state in a cyclic, irreversible manner and so cannot be the forward strokes of a proton pump. Again, the redox energy of dioxygen relative to cytochrome $c$ is largely lost going from $\mathrm{P} \rightarrow \mathrm{O}$ because the last two one-electron potentials of the oxidase are not far from those of cytochrome $c$ itself (Fig. 2). It is good sense that the cyclic functioning of a pump has highly energy-using initial steps and later relaxation steps driven by little energy loss.

How does this pump compare with other ion pumps, such as ATP-synthetase, $\mathrm{Na}^{+} / \mathrm{K}^{+}$ATPases and $\mathrm{Ca}^{2+}$ ATPases, and bacteriorhodopsin? In all these cases it has been supposed that cyclic helix/helix motions of proteins are part of the mechanical device in the pump. Moreover, it seems that the sites of energy input (here of ATP to the ion movements) are far in space from the gated channels, which are frequently considered to be constructed from anionic sites on helices within the membrane. The parallels with cytochrome oxidase are fairly close. Moreover in several cases where intermediates, such as phosphorylation of proteins, have been seen, the last steps, now loss of inorganic phosphate, act not in the pumping stroke but in relaxation, and release little energy.

It seems that several pumped movements of ions and possibly molecules in proteins in membranes have common features - helical protein structures, cycling conformation changes and readily distinguishable forward pumping and return steps where energy commitment is largely in the first steps.

R. J. P. Williams is the Napier Royal Society Research Professor in the Department of Inorganic Chemistry, University of Oxford, South Parks Road, Oxford OX1 3QR, UK.

1. Williams, R.J.P. J. theor. Biol. 1, 1-13 (1961)

Mitchell, P. Nature 191, 144-148 (1961)

3. Wikström, M. \& Saraste, M. in Bioenergetics (ed. Ernster. L.) Ch. 3 (Elsevier, Amsterdam, 1984)

4. Wikström, M. Nature 338, 775-778 (1989). $2823(1987)$

6. Williams, R.J.P. FEBS Lett. 226, 1 (1987).
5. Holm, L., Saraste, M. \& Wikström, M. EMBO J. 6. 2819

\section{Blow the fuse!}

LABORATORIES all round the world are trying to replicate the claimed electrolytic cold nuclear fusion from heavy water. Some may regard this as premature when the one crucial control experiment, the equivalent electrolysis of light water, has not yet been tried. But with his usual optimism, Daedalus has set DREADCO's electrochemists to forcing deuterium into palladium electrodes, and is determined to interpret any excess heat production as nuclear fusion, rather than some banal chemical or lattice effect.

Palladium has to take up a lot of deuterium before any heat is disengaged at all; but thereafter things seem to accelerate rapidly. This makes obvious sense: each site in the palladium lattice will take up one deuterium atom until all the sites are full. Further deuterium atoms will then enter already-occupied sites, and these closepacked pairs stand a chance of fusing together by quantum tunnelling. The energy released can, apparently, even melt the palladium.

But Daedalus goes further. He points out that as two nuclei approach each other, the chance of successful quantum tunnelling rises dramatically. So a sphere of deuteriumsaturated palladium would make an ideal implosion-style hydrogen bomb. At atmospheric pressure it would release a steady heat flux and an inconvenient but containable neutron flux. Surround it by shaped charges of high explosive and crush it suddenly to half its volume, and the result should be a splendid combined fusion explosion and neutron emission. Despite the deadly momentary neutron flux, this elegant bomb would release almost no enduring fallout: a valuable military advantage. Lacking a critical mass, it could also be made in usefully small sizes.

Daedalus is rather diffident about contributing to weapons research. In shifty self-defence he claims that nuclear physics is supported so lavishly by national governments only because it once produced a bomb. To keep the funds coming in, the subject really has to repeat the trick from time to time. In any case a pure, hygienic, noble-metal fusion weapon is a great ecological improvement on nasty poisonous and radioactive fission weapons, and should be supported enthusiastically by the increasingly militant 'green' political parties. But in more peaceful vein, Daedalus is also devising a fusion-powered watch. Each time its oscillating palladium hairspring flexes, deuterium fusion accelerates on its compressed face, and slows down on its tensioned face. The differential thermal expansion unwinds the spring; the inertia of the balance wheel drives it the other way, generating the opposite thermal gradient, and so on. A tiny charge of deuterium should run it for centuries. David Jones 